\title{
Belgeo
}

Revue belge de géographie

$4 \mid 2006$

Miscellaneous

\section{Bavoux J.J., Beaucire F., Chapelon I., Zembri P., Géographie des transports}

Coll. U, Paris, Armand Colin, 2005, 232 p.

\section{Frédéric Dobruszkes}

\section{OpenEdition}

\section{Journals}

Édition électronique

URL : http://journals.openedition.org/belgeo/11790

DOI : $10.4000 /$ belgeo. 11790

ISSN : 2294-9135

\section{Éditeur :}

National Committee of Geography of Belgium, Société Royale Belge de Géographie

\section{Édition imprimée}

Date de publication : 31 décembre 2006

Pagination : 500-501

ISSN : 1377-2368

\section{Référence électronique}

Frédéric Dobruszkes, «Bavoux J.J., Beaucire F., Chapelon I., Zembri P., Géographie des transports », Belgeo [En ligne], 4 | 2006, mis en ligne le 31 octobre 2013, consulté le 22 septembre 2020. URL : http://journals.openedition.org/belgeo/11790; DOl : https://doi.org/10.4000/belgeo.11790

Ce document a été généré automatiquement le 22 septembre 2020

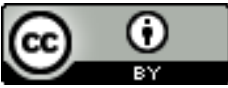

Belgeo est mis à disposition selon les termes de la licence Creative Commons Attribution 4.0 International. 


\title{
Bavoux J.J., Beaucire F., Chapelon I., Zembri P., Géographie des transports
}

\author{
Coll. U, Paris, Armand Colin, 2005, 232 p.
}

\author{
Frédéric Dobruszkes
}

\section{RÉFÉRENCE}

Bavoux J.J., Beaucire F., Chapelon I., Zembri P., Géographie des transports, Coll. U, Paris, Armand Colin, $232 \mathrm{p}$.

1 Il sort peu de livres de géographie des transports et l'on s'en voudrait donc de ne pas signaler celui-ci, sobrement intitulé Géographie des transports et rédigé par quatre géographes français parmi les plus connus de cette spécialité.

2 Le livre se décompose de fait en trois parties. Deux premiers chapitres traitent de la mobilité, l'un la (re)situant globalement et l'autre portant sur la vitesse selon une perspective plus historique. Suivent ensuite quatre chapitres (accessibilité, rugosité, réticularité, nodalité) développant plutôt des notions, concepts et outils d'analyse tout en passant en revue un certain nombre de rapports entre l'espace et les transports. Viennent alors trois chapitres relatifs à la "gouvernance des transports " (selon l'expression des auteurs) : planification, financement et environnement. Le livre se termine sous forme de questions passant en revue différents enjeux contemporains liés aux transports.

3 L'ouvrage apporte des concepts et éléments qui sont novateurs et/ou généralement rares chez les autres auteurs. Ainsi, la discussion sur l'évolution temporelle de l'accessibilité sur une journée est aussi convaincante qu'utile. L'habituelle contrainte de la distance est complétée par l'impact de l'altitude sur la performance des moteurs. Les apports de la théorie des graphes pour l'analyse des réseaux sont utilement résumés et débouchent sur l'originale mesure de la vulnérabilité des réseaux. Le vocabulaire peut parfois paraître ésotérique (citons par exemple les «interactions nodo-réticulaires ») 
mais décrit en fait des choses simples et compréhensibles, et qu'il est bon de mettre en avant.

4 On peut cependant regretter l'absence d'un vrai chapitre complet sur les grands facteurs conditionnant la géographie des réseaux d'infrastructures. Ceux-ci sont dispersés à travers le livre, ne sont pas complets et ne sont souvent pas accompagnés de cartes ou schémas qui illustreraient le propos. Sont ainsi traités trop rapidement, voire niés, le poids de l'histoire et du niveau de développement économique, le conditionnement par les systèmes urbains (une comparaison des réseaux TGV français et allemands, par exemple, aurait été ici très éclairante), les facteurs politiques (pouvant s'illustrer par exemple par le choix de réaliser le TGV Atlantique avant les TGV Nord et Méditerranée, ou le choix, non-acquis au début, de faire passer le TGV Nord par Lille) et géopolitiques (pourquoi pas au travers des tracés des oléoducs saoudiens visant à ne pas dépendre entièrement du Golfe persique pour l'évacuation des hydrocarbures?).

Dans le même ordre d'idée, l'ensemble des facteurs conditionnant la géographie des flux est présentée au début du livre, mais sur un ton général, sans illustration et sans intégrer tous les facteurs. Ainsi, les facteurs socio-spatiaux sont à peine évoqués, alors que des recherches anciennes de Chombart de Lauwe ${ }^{1}$ (qui montre l'espace pratiqué d'une jeune fille du XVI ${ }^{\mathrm{ème}}$ arrondissement, largement circonscrit aux quartiers aisés de l'ouest parisien) ou plus récentes de l'auteur de ces lignes ${ }^{2}$ (qui montre l'importance des clivages sociaux et religieux dans le recrutement d'écoles et donc la mobilité quotidienne des étudiants) en montrent l'importance à l'échelle urbaine ou régionale. C'est d'autant plus dommage que les sociologues qui ont récemment investi le champ des transports ${ }^{3}$ peinent à y intégrer les classes sociales, ou même les groupes sociaux, de par une posture post-moderniste et un discours philosophique et/ou ésotérique.

6 En outre, et cela semble être une ligne de conduite des auteurs, l'ouvrage ne dépasse guère les concepts, méthodes et faits généraux pour passer à une géographie des transports qui serait plus explicite et plus systématique. Le livre ne contient dès lors pas une géographie des transports ni en termes d'infrastructures, ni de flux, ni de feedback sur les structures spatiales. On ne trouve donc nulle part de cartographie des échanges mondiaux ou continentaux, des principaux noeuds de transport qui dominent la planète, de discussion sur les inégalités de desserte des continents, pays ou régions... Absence également d'un chapitre complet sur les transports urbains, et sur la géographie des télécommunications, que l'on peut légitimement voir comme relevant de la géographie des transports.

7 Enfin, même pour les thèmes traités par le livre, celui-ci n'est pas très riche en cartes ou schémas. Certains schémas sont difficiles à comprendre sans lire le texte, au contraire d'autres dont il faut signaler la clarté et l'efficacité pédagogique (nous pensons par exemple à la typologie des gares TGV, p. 102).

8 En conclusion, ce n'est pas tant le contenu du livre qui pose question que ce qui ne s'y trouve pas. On peut donc se demander si celui-ci n'aurait pas dû porter un titre tel que "Territoires et transports: méthodes et questions actuelles », auquel cas la plupart des critiques écrites ici n'auraient guère eu de sens. Sous cette réserve, l'ouvrage intéressera certainement les personnes, géographes et autres, intéressées par les transports. 


\section{NOTES}

1. Chombart de Lauwe P.-H. (1952), Paris et l'agglomération parisienne. Tome premier, L'espace social dans une grande cité, Paris, PUF, $109 \mathrm{p}$.

2. Dobruszkes F. (2005), « Déplacements et effets de la distance ou du temps : pour une prise en compte des surdéterminants sociaux", in Montulet B. et al. (dir.), Mobilités et temporalité, Bruxelles, Facultés universitaires Saint-Louis, coll. Travaux et Recherches, ${ }^{\circ}$ 51, pp. 67-88.

3. Voir par exemple Kaufmann V. et Montulet B. (2004), "Mobilités spatiales et fluidités sociales ", introduction de Mobilité, fluidités... libertés ?, Publications des Facultés universitaires Saint-Louis, Travaux et Recherches 46, pp. 9-21. 